OPEN ACCESS

Edited by:

Temel Tirkes,

Indiana University, United States

Reviewed by:

Beom Kyung Kim,

Yonsei University, South Korea

Rongxin Chen,

Fudan University, China

*Correspondence:

Pil Soo Sung

pssung@catholic.ac.kr

Si Hyun Bae

baesh@catholic.ac.kr

${ }^{+}$These authors have contributed equally to this work

Specialty section:

This article was submitted to

Cancer Imaging and

Image-directed Interventions,

a section of the journal

Frontiers in Oncology

Received: 29 August 2020

Accepted: 26 October 2020

Published: 19 November 2020

Citation:

Sung PS, Choi MH, Yang H, Lee SK, Chun HJ, Jang JW, Choi JY, Yoon SK,

Choi J-I, Lee YJ and Bae SH (2020)

Diffusion-Weighted Magnetic

Resonance Imaging in Hepatocellular

Carcinoma as a Predictor of a

Response to Cisplatin-Based Hepatic Arterial Infusion Chemotherapy.

Front. Oncol. 10:600233.

doi: 10.3389/fonc.2020.600233

\section{Diffusion-Weighted Magnetic Resonance Imaging in Hepatocellular Carcinoma as a Predictor of a Response to Cisplatin-Based Hepatic Arterial Infusion Chemotherapy}

Pil Soo Sung ${ }^{1,2 *}$, Moon Hyung Choi ${ }^{3 \dagger}$, Hyun Yang ${ }^{2,4}$, Soon Kyu Lee $^{2}$, Ho Jong Chun ${ }^{3}$, Jeong Won Jang ${ }^{1,2}$, Jong Young Choi ${ }^{1,2}$, Seung Kew Yoon ${ }^{1,2}$, Joon-ll Choi ${ }^{5}$, Young Joon Lee ${ }^{3}$ and Si Hyun Bae ${ }^{1,4 *}$

\footnotetext{
1 The Catholic University Liver Research Center, College of Medicine, The Catholic University of Korea, Seoul, South Korea, 2 Division of Gastroenterology and Hepatology, Department of Internal Medicine, College of Medicine, Seoul St. Mary's Hospital, The Catholic University of Korea, Seoul, South Korea, ${ }^{3}$ Department of Radiology, College of Medicine, Eunpyeong St. Mary's Hospital, The Catholic University of Korea, Seoul, South Korea, ${ }^{4}$ Division of Gastroenterology and Hepatology, Department of Internal Medicine, College of Medicine, Eunpyeong St. Mary's Hospital, The Catholic University of Korea, Seoul, South Korea, ${ }^{5}$ Department of Radiology, College of Medicine, Seoul St. Mary's Hospital, The Catholic University of Korea, Seoul, South Korea
}

This study aimed to identify the utility of diffusion-weighted magnetic resonance (MR) imaging with an apparent diffusion coefficient (ADC) map as a predictor of the response of hepatocellular carcinoma (HCC) to cisplatin-based hepatic arterial infusion chemotherapy (HAIC). We retrospectively evaluated 113 consecutive patients with Barcelona Clinical Liver Cancer (BCLC) stage B or C HCC, who underwent gadoxetic acid-enhanced and diffusion-weighted MR imaging. The appropriate cutoff for the pretreatment tumor-to-liver $A D C$ ratio was determined to be 0.741 . Of the 113 patients, 50 (44\%) presented with a pretreatment tumor-to-liver ADC ratio $<0.741$ (low group). Evaluation of the treatment response after 2-3 cycles of HAIC in these 50 patients revealed that 21 patients (42\%) experienced an objective response to HAIC. On the other hand, only 11 of the 63 patients (17\%) with a pretreatment tumor-to-liver ADC ratio $\geq 0.741$ (high group) showed an objective response. Thus, the objective response rate was significantly higher in the low group than in the high group $(P=0.006)$. Multivariate logistic regression analysis using parameters including perfusion alteration, percentage of non-enhancing portions, and pretreatment tumor-to-liver ADC ratio revealed that a pretreatment tumor-to-liver ADC ratio $<0.741$ (odds ratio $3.217 ; P=0.014$ ) was the sole predictor of an objective response to HAIC. Overall survival rates were significantly higher in patients with objective responses to HAIC than in those without objective responses ( $P=0.001$ by log-rank test). In conclusion, patients with $\mathrm{BCLC}$ stage $\mathrm{C}$ or $\mathrm{C} \mathrm{HCC}$ with a pretreatment tumor-to-liver $\mathrm{ADC}$ ratio $<0.741$ showed a favorable intrahepatic response to cisplatin-based HAIC. 
Therefore, diffusion-weighted MR imaging can play a critical role as a predictor of response to cisplatin-based HAIC in unresectable HCC.

Keywords: hepatocellular carcinoma, hepatic arterial infusion chemotherapy, magnetic resonance, diffusion restriction, objective response

\section{INTRODUCTION}

Hepatocellular carcinoma (HCC) is the fourth most common cause of malignancy-related death worldwide (1). A considerable number of patients with advanced HCC receive only palliative treatments in East Asian countries, where hepatitis B virus (HBV) infection is prevalent and accounts for more than $70 \%$ of the patients (2). To enhance survival outcomes, sorafenib and lenvatinib are usually administered in cases of advanced HCC with portal vein tumor thrombus (PVTT) or extrahepatic metastasis. However, these drugs only have modest treatment responses and may even have notable side effects $(3,4)$. Furthermore, the latest immune checkpoint inhibitor monotherapy did not demonstrate increased survival compared with sorafenib in patients with unresectable HCC (5).

Barcelona Clinical Liver Cancer (BCLC) stage B or C HCC cases with high intrahepatic tumor burden can alternatively be treated through hepatic arterial infusion chemotherapy (HAIC), whereby the drug is administered directly through a port inserted into the liver. HAIC enables higher drug concentration in intrahepatic tumors with minimal systemic adverse effects (2). There is research evidence that, compared to sorafenib, both the objective response and survival outcomes are improved when advanced HCC is treated through $\operatorname{HAIC}(2,6,7)$. Moreover, recent studies demonstrated that significant reduction of the intrahepatic tumor by HAIC in HCC with PVTT and/or extrahepatic metastases led to better survival outcomes than no reduction of the intrahepatic tumor burden $(2,8)$. Therefore, in advanced HCC, it is crucial to identify patients who will potentially benefit from HAIC before start the treatment.

A non-invasive diagnosis of HCC is established by a characteristic radiological findings of arterial phase hyperenhancement (APHE) and portal venous or delayed phase "washout" on contrast-enhanced computed tomography (CT) or magnetic resonance imaging (MRI) (9). Recently, a hepatocyte-specific contrast agent, gadoliniumethoxybenzyl-diethylenetriamine penta-acetic acid (Gd-EOBDTPA, gadoxetic acid), has been recognized as the critical tool for the detection of early HCCs. Moreover, diffusion-weighted imaging (DWI) obtained during MR examination was reported to estimate the biological behavior of HCC $(10,11)$. In general, DWI depends on the information of the diffusivity of water molecules, reflecting the cellularity of the tumors (12). DWI has a potential for use in various liver diseases (13). It can be used as a biomarker for liver fibrosis, HCC detection, and predicting responses to anticancer therapies (14). DWI is an attractive technique in liver diseases because it may add qualitative and quantitative information to conventional MRI sequences and it can be easily performed (13).

Evaluation of HCC treatment responses based on the detection of features in DWI MR has been undertaken by several studies. Previous reports showed that pretreatment apparent diffusion coefficient (ADC) of HCC can be predictive of response to transarterial chemoembolization (TACE) (15, 16). Moreover, ADC change relative to baseline (ADC ratio) 1 month after TACE was an independent predictor of progression-free survival in HCC (17). However, there are no reports that describe the association between various MR parameters and responses to HAIC in HCC. In this study, we aimed to identify the utility of various MRI findings and DWI with an ADC map as a predictor of the intrahepatic response of HCC to cisplatin-based HAIC.

\section{METHODS}

\section{Study Design and Population}

Ethical approval was obtained from the Institutional Review Board of Seoul St. Mary's Hospital (KC19RESI0912). A diagnosis of HCC was confirmed in every enrolled patient by the updated international guidelines $(9,18,19)$. HCC cases with PVTT or infiltrative tumors are occasionally treated with HAIC rather than sorafenib or lenvatinib in the researcher's institution. The medical records of all cases that received an HCC diagnosis between January 2010 and December 2017 were reviewed by experienced hepatologists. The survival data of the patients continued to be followed up until December 2019. The inclusion criteria of this study were as follows: unresectable HCC cases with Barcelona Clinical Liver Cancer (BCLC) stage B or C undergoing HAIC monotherapy, age range of $20-80$ years, Child-Pugh class A or B, Eastern Cooperative Oncology Group (ECOG) performance status of less than 2, lack of indication of bone marrow inhibition (white blood cell $\geq 3000 / \mu \mathrm{L}$, hemoglobin $\geq 8 \mathrm{~g} / \mathrm{dL}$, and platelet count $\geq$ $7.5 \times 10^{4} / \mu \mathrm{L}$ ), normal renal function with levels of serum creatinine $<$ $2.0 \mathrm{mg} / \mathrm{dL}$, diffusion-weighted, contrast-enhanced MR imaging before treatment, and response evaluation at least after 2 cycles of treatment. The study did not include cases in which HAIC was undertaken after sorafenib administration. Patients without response evaluation or with combination treatments (HAIC + sorafenib or HAIC + radiation therapy) were excluded from the analyses. Finally, 113 patients were enrolled in this study (Figure 1).

\section{Diagnosis of HCC}

Multiphasic CT, MRI, biochemical analysis of alpha-fetoprotein (AFP), and additional biomarkers provided diagnostic information for HCC. The modified RECIST criteria were used to assess therapeutic response (3). Tumors without arterial enhancement of the target lesions were determined to be in complete response (CR). Partial response (PR) was defined as a $30 \%$ reduction in the sum of viable target lesion diameters. Progressive disease (PD) was identified if augmentation of at least $20 \%$ was noted in the total viable target lesion diameters. If the findings were outside these definitions, the tumor response was considered a stable disease 


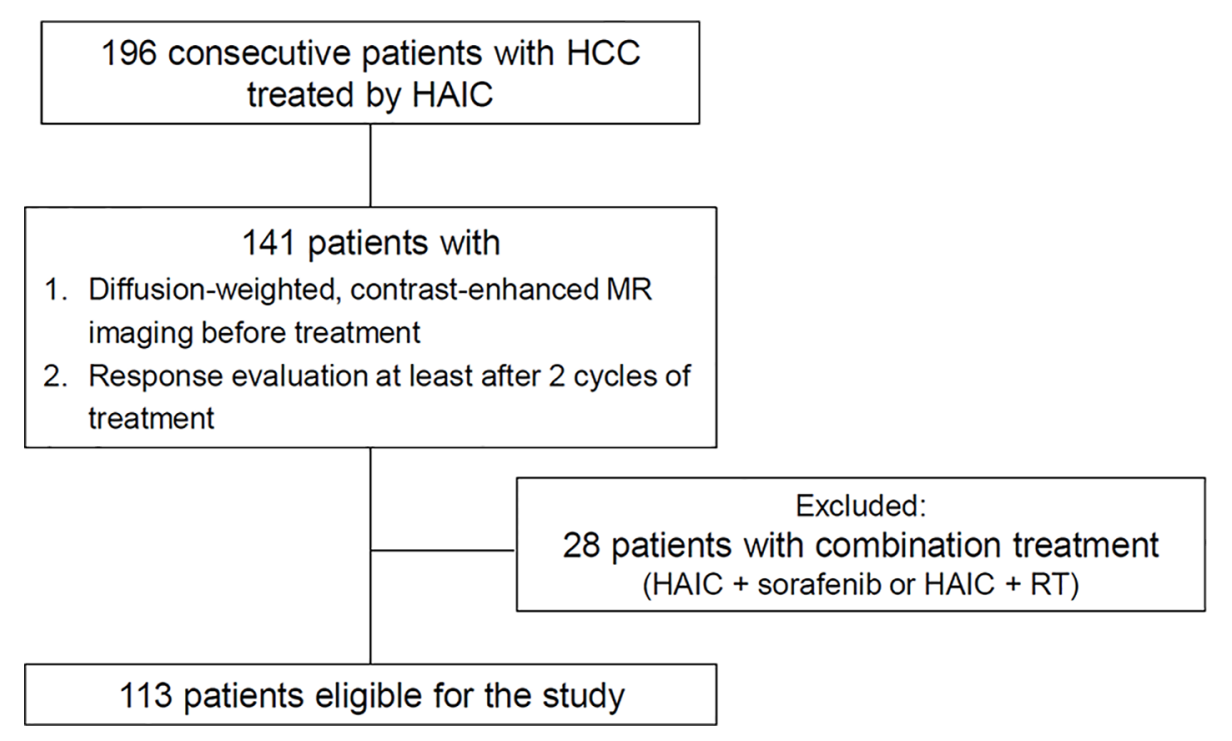

FIGURE 1 | Study population.

(SD). RECIST rather than mRECIST was used in cases of infiltrative HCCs because this type is classified by mRECIST as a non-target lesion. Likewise, during the assessment of tumor response, PVTT was not included because it was classified by mRECIST as a nontarget lesion.

The Vp stages were employed to categorize the PVTT. Tumor invasion distal to the second portal vein branch was categorized as Vp1, tumor invasion of the second portal vein branch was categorized as $\mathrm{Vp} 2$, tumor invasion of the first portal vein branch was categorized as $\mathrm{Vp} 3$, and tumor thrombus occurrence in the main portal vein trunk or a branch of the portal vein contralateral to the main affected lobe was categorized as Vp4 (2).

\section{HAIC Protocol}

The specific protocol of cisplatin-based HAIC has been described previously (2). Every HAIC process was conducted by two or more interventional radiologists with more than five years of experience. Two chemotherapeutic drugs were infused through the chemoport inserted into the femoral artery, 5-fluorouracil (5FU) $\left(500 \mathrm{mg} / \mathrm{m}^{2}\right)$ for three days and cisplatin $\left(60 \mathrm{mg} / \mathrm{m}^{2}\right)$ on the second day. In cases where the disease did not progress or the therapy did not have severe complications, HAIC was repeated at the interval of 4-6 weeks. The Child-Pugh categorization was applied at every cycle to assess hepatic function, while follow-up multiphasic CT or MRI was applied after 2-3 therapy cycles to assess the response to therapy.

\section{Qualitative and Quantitative Analyses of MR Imaging}

A 3-T MR system (Verio, Siemens Healthcare, Erlangen, Germany) alongside a phased array coil with 8 channels serving as the receiver coil was employed for MR imaging. Breath-hold half Fourier Acquisition single-shot turbo spin echo, respiratory-triggered fast spin echo T2-weighted image with fat suppression and 3D T1weighted in- and opposed-phase gradient echo with two-point Dixon reconstruction were obtained as previously described (16). Meanwhile, contrast-enhanced study was performed using fatsuppressed 3D spoiled gradient-echo volume interpolated breathhold examinations. After acquisition of unenhanced images, GdEOB-DTPA was injected with a dosage of $0.1 \mathrm{~mL} / \mathrm{kg}$ body weight and a flow rate of $1 \mathrm{~mL} / \mathrm{s}$ through the antecubital vein followed by a 20-mL saline flush. Arterial phase (30- to 35-second delay), portal venous phase (65- to 85-second delay), transitional phase (3-minute delay), and hepatobiliary phase (HBP) (20-minute delay) were acquired as previously described (20). DWI with echo planar imaging using b values of $0,50,500$, and $800 \mathrm{~s} / \mathrm{mm}^{2}$ were obtained and ADC maps were automatically generated using DWI with $\mathrm{b}$ values of 0 and $800 \mathrm{~s} / \mathrm{mm}^{2}$ (20). MR imaging sequences and parameters are presented in Table $\mathbf{1}$.

In this study, several radiologists discussed the methods to quantify the ADC values of tumors, background livers, and spleen. They made agreements how to quantitate ADC values. The data from the patients, which were used in the analyses, were obtained from one experienced radiologists (more than 10 years of experience in abdominal radiology) among the involved radiologists. She recorded the number of tumors (single or multiple), the largest diameter on axial and coronal images, presence of portal vein tumor thrombus, proportion of nonenhancing portion $(<50 \%$ or $\geq 50 \%)$, perfusion alteration, targetoid enhancement, blood product in tumor, fatty change in tumor, diffusion restriction, tumor signal intensity on arterial phase, and homogeneous enhancement on arterial phase. Definition of the most imaging findings were based on the Liver Imaging - Reporting and Data System (LI-RADS). Perfusion alteration is change from the usual blood supply in the liver parenchyma and we evaluated the presence of regional perfusion alteration near the tumor. Targetoid 
enhancement is target-like imaging morphology with concentric arrangement of internal components. Blood product is seen as high signal intensity on T1 weighted images of MRI. Intra-lesion fat is increased fat within the tumor and we evaluated it on in and opposed phases of MRI.

APHE is enhancement in arterial phase and enhancing part must be higher than liver parenchyma. So, signal intensity or density of tumor on arterial phase was compared to the liver parenchyma and divided into three categories (higher, similar and lower). Arterial phase enhancement that is most pronounced in periphery (rim APHE) is atypical feature of HCC, homogeneity of arterial enhancement was evaluated whether the arterial enhancement in the tumor was prominent in periphery or not.

Quantitative measurement was undertaken regarding ADC values in lesions and circumscribing healthy parenchyma. Delineation of a region of interest was done on ADC maps for both healthy liver parenchyma and HCCs, steering clear of necrotic and cystic zones, artifacts, and blood vessels within the liver as far as manageable. The regions of interest were drawn with similar size (3 $\mathrm{cm}^{2}$ ) in both of tumors and healthy parenchyma and peripheral portion of the tumor was not included because of frequent partial volume artifact. The region of interest was drawn in the largest tumor in the patients with multiple tumor (20). Ratio of pretreatment tumor-to-liver ADC (tumor ADC/liver ADC) was calculated in each patient.

\section{Statistical Analysis}

Statistical analysis was performed using SPSS version 26 software (IBM Corp., Armonk, NY, USA). The chi-square test was used to evaluate discrete variables from the two cohorts. An independent ttest was employed to compare continuous variables between the two groups. To determine the optimal cut-off value in discriminating objective responses by mRECIST, receiver operating characteristic (ROC) curves were generated for pretreatment tumor-to-liver ADC ratio (Supplementary Figure 1). Sensitivity, and specificity were calculated by the optimal cut-off value. Statistical significance was defined as $P<0.05$. The KaplanMeier technique was adopted to estimate the overall survival (OS), and the log-rank test was applied for OS comparison. Determinants of objective responses were identified by conducting multivariate analysis alongside a logistic regression model.

\section{RESULTS}

\section{Baseline Characteristics}

The baseline clinical characteristics of the enrolled patients are listed in Table 2. We divided all included patients into two groups according to the pretreatment tumor-to-liver $\mathrm{ADC}$ ratio. The cutoff value of the pretreatment tumor-to-liver ADC ratio (0.741) was determined by the receiver operating characteristic (ROC) curve. The patients were divided into two groups: patients with a pretreatment tumor-to-liver ADC ratio < 0.741 ( $\mathrm{n}=50$, low group) and a pretreatment tumor-to-liver $\mathrm{ADC}$ ratio $\geq 0.741$ ( $\mathrm{n}=$ 63 , high group). There were no differences in sex and etiology of HCC between the two groups, although patients in the low group tended to be younger (Table 2). There were no statistical differences in the maximal diameter, tumor number, and the presence of PVTT between the two groups. There were no differences in BCLC stages between the two groups. Most of the patients in both groups were classified as BCLC-C stages. Liver function measured by ChildPugh class were not significantly different between two groups. A considerable number of patients in both groups received other modalities of treatment (TACE, liver resection, RFA, or sorafenib) before HAIC. Regarding tumor markers, there was no statistical difference in serum AFP levels between the two groups. Before HAIC, the mean tumor ADC (unit, $1.10 \pm 0.29 \times 10^{-3} \mathrm{~mm}^{2} / \mathrm{s}$ ) of the high group was not significantly different from that of the low group (unit, $1.10 \pm 0.31 \times 10^{-3} \mathrm{~mm}^{2} / \mathrm{s}$ ), although the mean pretreatment tumor-to-liver ADC ratio was significantly lower in the low group $(P<0.001)$ (Table 2). Moreover, the mean pretreatment tumor-tospleen ADC ratio was also significantly lower in patients in the low group $(P<0.001)$, although spleen ADC values were not significantly different between two groups. For the other parameters detected in MR imaging (the amount of the nonenhancing portion, the presence of perfusion alteration, the presence of targetoid enhancement, the presence of blood product in the tumor, the presence of fatty change in the tumor, and the tumor signal on arterial phase), there were no statistical differences in these parameters between the low and high groups.

\section{Intrahepatic Response According to the Pretreatment ADC Tumor-to-Liver Ratio}

As indicated in Table 2, the optimal intrahepatic response to therapy was evaluated based on mRECIST following 2-3 HAIC

TABLE 1 | MR imaging sequences and parameters.

\begin{tabular}{|c|c|c|c|c|c|}
\hline \multirow[t]{2}{*}{ Parameters } & \multicolumn{5}{|c|}{ Sequence } \\
\hline & HASTE T2WI & Fast Spin Echo T2WI & T1-weighted in/opposed phase & T1-weighted 3D GRE & DWI \\
\hline $\mathrm{TR}(\mathrm{ms})$ & $600-1000$ & $2000-6000$ & $170-220$ & $2.8-3.5$ & $3500-4200$ \\
\hline $\mathrm{TE}(\mathrm{ms})$ & $80-140$ & $100-140$ & 2.6/1.3 & $1-1.2$ & $40-50$ \\
\hline Flip angle $\left(^{\circ}\right)$ & 138 & $150-160$ & $50-70$ & 11 & $90 / 180$ \\
\hline Slice thickness (mm) & 6 & 6 & 6 & 2 & 8 \\
\hline Reconstruction interval (mm) & 6 & 6 & 6 & 2 & 8 \\
\hline Acquisition matrix & $320-400 \times 150-180$ & $380-450 \times 180-220$ & $250-300 \times 120-170$ & $256 \times 156$ & $140-160 \times 90-120$ \\
\hline Signal averages & 1 & 1 & 1 & 1 & 5 \\
\hline b-values (s/mm²) & $\mathrm{N} / \mathrm{A}$ & N/A & $\mathrm{N} / \mathrm{A}$ & NA & $0,50,500$ \\
\hline
\end{tabular}

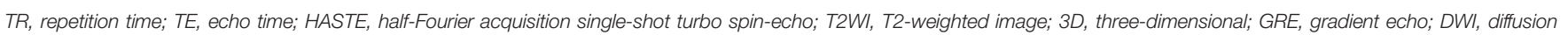
weighted image; N/A, not assessed. 
TABLE 2 | Clinical parameters of study patients.

\begin{tabular}{|c|c|c|c|c|c|}
\hline \multirow[t]{3}{*}{ Variables } & \multirow{2}{*}{\multicolumn{2}{|c|}{$\frac{\text { ADC ratio }<0.741}{(n=50)}$}} & \multirow{2}{*}{\multicolumn{2}{|c|}{$\frac{A D C \text { ratio } \geq 0.741}{(n=63)}$}} & \multirow[t]{3}{*}{$P$} \\
\hline & & & & & \\
\hline & No. & $\%$ & No. & $\%$ & \\
\hline \multicolumn{5}{|l|}{ Age } & 0.02 \\
\hline$<60$ & 36 & 72 & 31 & 49 & \\
\hline$\geq 60$ & 14 & 28 & 32 & 51 & \\
\hline \multicolumn{5}{|l|}{ Sex } & 0.074 \\
\hline Male & 44 & 88 & 47 & 75 & \\
\hline Female & 6 & 12 & 16 & 25 & \\
\hline \multicolumn{5}{|l|}{ Etiology } & 0.096 \\
\hline HBV & 46 & 92 & 46 & 73 & \\
\hline $\mathrm{HCV}$ & 1 & 2 & 7 & 11 & \\
\hline Alcohol & 1 & 2 & 6 & 10 & \\
\hline $\mathrm{HBV}+\mathrm{HCV}$ & 0 & 0 & 1 & 2 & \\
\hline Others & 2 & 4 & 3 & 5 & \\
\hline \multicolumn{5}{|l|}{ BCLC stage } & 0.533 \\
\hline A & 0 & 0 & 0 & 0 & \\
\hline $\mathrm{B}$ & 6 & 12 & 5 & 8 & \\
\hline $\mathrm{C}$ & 44 & 88 & 58 & 92 & \\
\hline \multicolumn{5}{|l|}{ Serum AFP } & 0.257 \\
\hline$<1000$ ng/mL & 19 & 38 & 31 & 49 & \\
\hline$\geq 1000$ ng/mL & 31 & 62 & 32 & 51 & \\
\hline \multicolumn{5}{|l|}{ Tumor maximal diameter } & 0.437 \\
\hline$<10 \mathrm{~cm}$ & 17 & 34 & 27 & 43 & \\
\hline$\geq 10 \mathrm{~cm}$ & 33 & 66 & 36 & 57 & \\
\hline \multicolumn{5}{|l|}{ Tumor number } & 0.703 \\
\hline Single & 23 & 46 & 26 & 41 & \\
\hline Multiple & 27 & 54 & 37 & 59 & \\
\hline \multicolumn{5}{|l|}{$\begin{array}{l}\text { Portal vein tumor } \\
\text { thrombus }\end{array}$} & 0.829 \\
\hline Vpo, 1, 2 & 12 & 24 & 17 & 27 & \\
\hline Vp3, 4 & 38 & 76 & 46 & 73 & \\
\hline \multicolumn{5}{|l|}{ Extrahepatic metastasis } & 1 \\
\hline yes & 11 & 22 & 13 & 21 & \\
\hline no & 39 & 78 & 50 & 79 & \\
\hline \multicolumn{5}{|l|}{ Child-Pugh class } & 0.314 \\
\hline A & 27 & 54 & 38 & 60 & \\
\hline B & 23 & 46 & 25 & 40 & \\
\hline \multicolumn{6}{|l|}{ Previous treatment } \\
\hline TACE & 20 & 40 & 26 & 52 & \\
\hline RFA & 3 & 6 & 5 & 10 & \\
\hline TARE & 1 & 2 & 3 & 6 & \\
\hline Liver resection & 2 & 4 & 4 & 8 & \\
\hline Sorafenib & 1 & 2 & 3 & 6 & \\
\hline \multicolumn{5}{|l|}{ Non-enhancing portion } & 0.005 \\
\hline$<50 \%$ & 26 & 52 & 49 & 78 & \\
\hline$\geq 50 \%$ & 24 & 48 & 14 & 22 & \\
\hline \multicolumn{4}{|l|}{ Perfusion alteration } & 0 & 0.126 \\
\hline no & 26 & 52 & 42 & 67 & \\
\hline yes & 24 & 48 & 21 & 33 & \\
\hline Targetoid enhancement & & & & & 0.599 \\
\hline no & 44 & 88 & 53 & 84 & \\
\hline yes & 6 & 12 & 10 & 16 & \\
\hline $\begin{array}{l}\text { Blood product in tumor } \\
\text { (T1) }\end{array}$ & & & & & 0.852 \\
\hline no & 26 & 52 & 34 & 54 & \\
\hline yes & 24 & 48 & 29 & 46 & \\
\hline Fatty change in tumor & & & & & 1 \\
\hline no & 44 & 88 & 56 & 89 & \\
\hline yes & 6 & 12 & 7 & 11 & \\
\hline $\begin{array}{l}\text { Mean tumor ADC } \\
\text { (unit, } \times 10^{-3} \mathrm{~mm}^{2} / \mathrm{s} \text { ) }\end{array}$ & 1.10 & & 1.10 & & 1 \\
\hline
\end{tabular}

(Continued)
TABLE 2 | Continued

\begin{tabular}{|c|c|c|c|c|c|}
\hline \multirow[t]{3}{*}{ Variables } & \multirow{2}{*}{\multicolumn{2}{|c|}{$\begin{array}{c}\text { ADC ratio }<0.741 \\
(n=50)\end{array}$}} & \multirow{2}{*}{\multicolumn{2}{|c|}{$\begin{array}{c}\text { ADC ratio } \geq 0.741 \\
(n=63)\end{array}$}} & \multirow[t]{3}{*}{$P$} \\
\hline & & & & & \\
\hline & No. & $\%$ & No. & $\%$ & \\
\hline $\begin{array}{l}\text { Mean liver ADC } \\
\text { (unit, } \times 10^{-3} \mathrm{~mm}^{2} / \mathrm{s} \text { ) }\end{array}$ & \multicolumn{2}{|c|}{$1.54 \pm 0.35$} & \multicolumn{2}{|c|}{$1.26 \pm 0.31$} & 0.001 \\
\hline $\begin{array}{l}\text { Mean spleen ADC (unit, } \\
\times 10^{-3} \mathrm{~mm}^{2} / \mathrm{s} \text { ) }\end{array}$ & \multicolumn{2}{|c|}{$1.05 \pm 0.24$} & \multicolumn{2}{|c|}{$1.06 \pm 0.19$} & 0.757 \\
\hline $\begin{array}{l}\text { Mean tumor-to-liver } \\
\text { ADC ratio }\end{array}$ & \multicolumn{2}{|c|}{$0.63 \pm 0.10$} & \multicolumn{2}{|c|}{$0.98 \pm 0.23$} & 0.001 \\
\hline $\begin{array}{l}\text { Mean tumor-to-spleen } \\
\text { ADC ratio }\end{array}$ & \multicolumn{2}{|c|}{$0.94 \pm 0.25$} & \multicolumn{2}{|c|}{$1.16 \pm 0.27$} & 0.001 \\
\hline Diffusion restriction & & & & & 0.787 \\
\hline no & 8 & 16 & 8 & 13 & \\
\hline yes & 42 & 84 & 55 & 87 & \\
\hline $\begin{array}{l}\text { Tumor signal on arterial } \\
\text { phase }\end{array}$ & & & & & 0.662 \\
\hline higher than parenchyma & 45 & 90 & 56 & 89 & \\
\hline $\begin{array}{l}\text { similar to liver } \\
\text { parenchyma }\end{array}$ & 2 & 4 & 4 & 6 & \\
\hline $\begin{array}{l}\text { lower than liver } \\
\text { parenchyma }\end{array}$ & 3 & 6 & 3 & 5 & \\
\hline $\begin{array}{l}\text { Homogenous } \\
\text { enhancement }\end{array}$ & & & & 0 & 0.014 \\
\hline no & 6 & 12 & 20 & 32 & \\
\hline yes & 44 & 88 & 43 & 68 & \\
\hline $\begin{array}{l}\text { Median HAIC session } \\
\text { number }\end{array}$ & \multicolumn{2}{|c|}{$5.0 \pm 3.3$} & \multicolumn{2}{|c|}{$4.3 \pm 3.1$} & 0.297 \\
\hline Response to HAIC & & & & & 0.006 \\
\hline CR+PR & 21 & 42 & 11 & 17 & \\
\hline SD+PD & 29 & 58 & 52 & 83 & \\
\hline
\end{tabular}

HAIC, Hepatic arterial infusion chemotherapy; HBV, hepatitis B virus; $H C V$, hepatitis $C$ virus; BCLC, Barcelona Clinical Liver Cancer; AFP, alpha-fetoprotein; TACE, transarterial chemoembolization; RFA, radiofrequency ablation; TARE, transarterial radioembolization; ADC, apparent diffusion coefficient.

Significant variables between two groups are in bold characters.

cycles. In the low group, the number of patients who displayed CR or PR was 21 (42\%) and SD or PD was 29 (58\%). In the high group, the number of patients who displayed CR or PR was 11 $(17 \%)$ and SD or PD was $52(83 \%)$. There was a statistical difference between the objective response rate between the low and the high group $(P=0.006)$. Figure 2 shows that the pretreatment tumor-to-liver ADC ratio of patients with objective responses was significantly lower than that of patients without objective responses $(P<0.01)$. OS were significantly higher in patients with objective responses to HAIC than in those without objective responses $(P=0.001$ by log-rank test, Supplementary Figure 2A). However, there was no significant difference in OS (Supplementary Figure 2B) and progressionfree survival (Supplementary Figure 2C) between the patients with high pretreatment tumor-to-liver ADC ratio and those with low pretreatment tumor-to-liver ADC ratio by log-rank test.

\section{Factors Affecting Responses to HAIC}

Table 3 delineates the factors affecting the responses to HAIC. Variables included in the logistic regression were as follows: age $<60$ years, male sex, maximal tumor diameter $<10 \mathrm{~cm}$, presence of PVTT as Vp0 to Vp2, AFP lower than 1,000 ng/mL, nonenhancing portion of the tumor less than $50 \%$, the presence of 


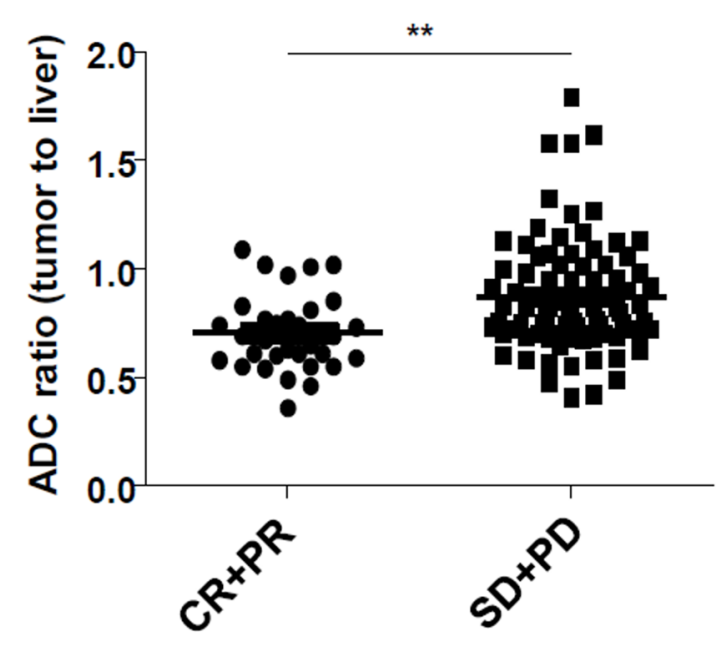

\section{Response to HAIC}

FIGURE 2 | Pretreatment tumor-to-liver ADC ratio according to the response to HAIC. ${ }^{* *} P<0.01$.

perfusion alteration, and the pretreatment tumor-to-liver ADC ratio less than 0.741 (Table 3 ). Among all the factors, the pretreatment tumor-to-liver ADC ratio was the only factor that had a significant effect on the objective responses to cisplatinbased HAIC (odds ratio: 3.217, 95\% confidence interval: 1.2648.187, $P=0.014$ ) (Table 3).

Figure 3 shows the MR imaging of a representative patient case having HCC with strong diffusion restriction and excellent response to HAIC. For the patient, the main tumor was located in the main portal vein, and the pretreatment tumor-to-liver ADC ratio was 0.36 . After 5 cycles of cisplatin-based HAIC, there was no viable tumor with diffusion restriction (Figure 3). The patient underwent subsequent liver transplantation after the achievement of CR by HAIC. Explant histology showed no viable tumor and complete pathologic response to HAIC.

TABLE 3 | Factors affecting the responses to HAIC.

\begin{tabular}{|c|c|c|}
\hline \multirow[t]{2}{*}{ Variables } & \multicolumn{2}{|c|}{$\begin{array}{c}\text { Logistic regression analysis for } \\
\text { objective response }\end{array}$} \\
\hline & $P$ & OR (95\% Cl) \\
\hline Age $(<60)$ & 0.374 & $0.643(0.244-1.699)$ \\
\hline Sex (male) & 0.423 & $1.662(0.479-5.761)$ \\
\hline Size $(<10 \mathrm{~cm})$ & 0.683 & $1.217(0.473-3.131)$ \\
\hline Portal vein tumor thrombus (Vp0 to Vp2) & 0.789 & $0.862(0.290-2.566)$ \\
\hline $\operatorname{AFP}(<1000 \mathrm{ng} / \mathrm{mL})$ & 0.962 & $1.023(0.404-2.593)$ \\
\hline Non-enhancing portion (< 50\%) & 0.559 & $0.737(0.265-2.049)$ \\
\hline Perfusion alteration (present) & 0.388 & $0.654(0.250-1.715)$ \\
\hline Pretreatment tumor-to-liver ADC ratio & 0.014 & 3.217 (1.264-8.187) \\
\hline
\end{tabular}

HAIC, Hepatic arterial infusion chemotherapy; AFP, alpha fetoprotein; ADC, apparent diffusion coefficient; OR, odds ratio; $\mathrm{Cl}$, confidence interval.

Significant variable in regression analysis is in bold characters.

\section{DISCUSSION}

Advanced HCC usually shows poor prognosis, with the aim of treatment being limited to extending life and at the same time preserving the hepatic reserve. BCLC stage B or C HCC with high intrahepatic tumor burden has typically been treated with the multikinase inhibitor sorafenib or lenvatinib (21). However, these drugs have been shown to improve survival only slightly. Moreover, when HBV is the cause of HCC, the prevalence of PVTT and more aggressive tumor features is higher than when other etiologies are the causes (2). Recent work by our group has demonstrated that survival outcomes in some advanced HCC cases may be improved dramatically by HAIC because of the substantial reduction of the intrahepatic tumor burden, even in cases with Vp 3/4 PVTT or extrahepatic metastases (2). Therefore, it is critical to identify the patients who will potentially benefit from HAIC. In this study, we suggest that decreased pretreatment tumor-to-liver ADC ratio may be a marker of an objective response to cisplatin-based HAIC. We compared the cutoff value of ADC tumor-to-liver ratio (0.741) in this study with those of prior studies in patients HCC. There were a few studies that described the prognostic significance of tumor-toliver ADC ratio in HCC. In one study, the mean tumor-to-liver ADC ratio was $1.13 \pm 0.63$. The study included patients with pathologically confirmed HCC, and the ADC ratio was neither associated with tumor size or differentiation grade. In another study, lower tumor-to-liver ADC ratio (cutoff: 0.820) was a significant factor to predict CK19-positive HCC. Our cutoff level (0.741) is similar with that in the latter study (0.820). Because HAIC is usually performed in patients with advanced HCC, the cutoff value of our study may be lower than those of other studies, reflecting higher cellularity of the tumors.

DWI was used for predicting the responses to various local and systemic therapies in patients with HCC (22). For TACE, a significant increase in the mean ADC of the tumors with a simultaneous reduction in the intra-tumoral enhancement was reported in treated tumors $(16,17)$. For radioembolization, a previous study reported a modest ADC increases post-treatment (20). For the systemic treatment, a pilot study reported that a significant increase in perfusion fraction was noted in sorafenib responders, although overall ADC was not significantly altered between responders and non-responders $(23,24)$.

DWI is now used in most of the cancers to predict treatment responses and to distinguish different tumor grades (12). For instance, patients with breast cancer and a low pretreatment ADC tended to respond better to neoadjuvant chemotherapy (2). Recent reports demonstrated that DWI helps distinguishing early HCCs from regenerative nodules in cirrhotic livers (22, 25). Moreover, DWI predicted the pathologic grade of HCC because there was an inverse correlation between tumor grades and $\operatorname{ADC}$ values $(13,26,27)$. For patients treated with cisplatinbased HAIC, this strategy will also provide benefits to patients. Despite the known chemoresistance of HCC to cytotoxic drugs such as cisplatin $(17,28)$, there are certainly a group of patients that show dramatic responses to this treatment (29). The reason there is a group of patients who show objective responses to this treatment will be identified when detailed multi-omics analyses are performed. Previous reports demonstrated that downregulated 


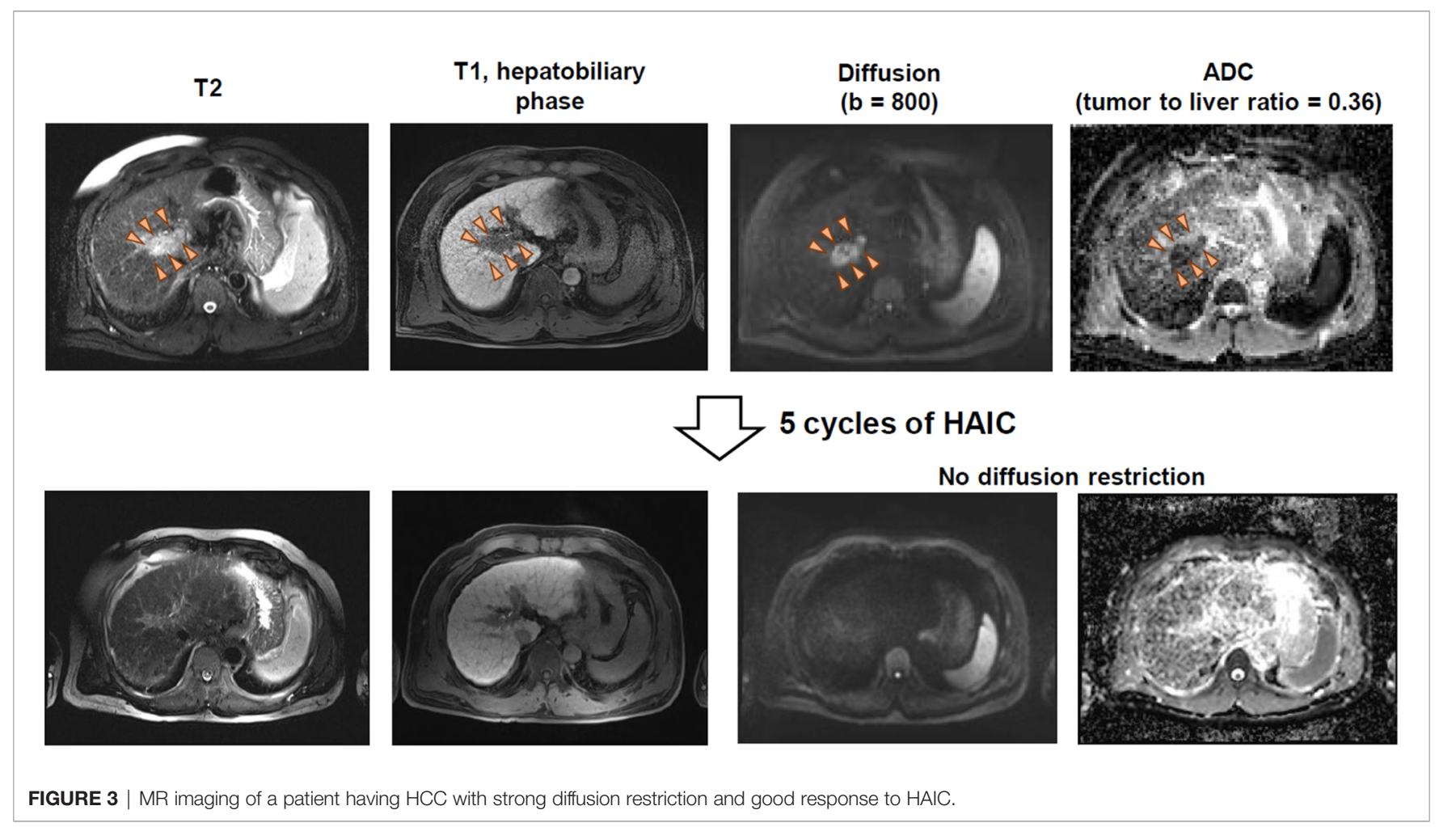

expression of specific genes may render susceptibility to cisplatin in HCC cell lines $(17,28)$. This suggests that patients with downregulation of these genes in their tumors may show a good response to cisplatin-based HAIC. The molecular-radiologic correlation regarding cisplatin sensitivity in $\mathrm{HCC}$ requires further investigation.

There are a number of shortcomings to this study. One shortcoming is that the study was conducted in one institution, so there is a possibility of selection bias. Another shortcoming is the insufficient number of cases recruited. Moreover, liver fibrosis/cirrhosis can lower the ADC values of the liver parenchyma on MRI. Therefore, the pretreatment tumor-toliver $\mathrm{ADC}$ ratio could be affected the degree of liver fibrosis in this study. Moreover, PVTT is not measurable in mRECIST criteria and most of the patients with good responses to HAIC in this study had PVTT, which might have caused the tumor burden measured by mRECIST not to be associated with HAIC responses. A cohort study with a larger number of patients with more stratified analyses should be performed. On the other hand, this is the first study to identify imaging biomarkers of HAIC in advanced HCC. Although comparable analyses were conducted on cases receiving sorafenib treatment during the same period, statistical analyses were not possible because only five of the over 250 cases displayed intrahepatic objective responses following sorafenib treatment.

In a recent clinical trial, lenvatinib was non-inferior to sorafenib in terms of OS in patients with unresectable HCC and caused a considerable decrease in the tumor burden when patients were responsive to the drug (30-32). Therefore, the combined use of lenvatinib plus cisplatin-based HAIC may show the synergistic anti-cancer effects in advanced HCC. A future prospective clinical trial of lenvatinib plus cisplatin-based HAIC vs. lenvatinib only may show promising results in combination treatment. Investigation of the roles of DWI and contrastenhanced MRI in lenvatinib plus HAIC will also be an area for interesting research.

HCC is a typical example of malignancy associated with nonresolving inflammation (33-35). However, HCC is recognized as an immune-tolerant malignancy (36). Only $14 \%$ to $18 \%$ of patients who receive pembrolizumab or nivolumab monotherapy demonstrate objective tumor responses (37-39). In HCC, immune heterogeneity is characteristic of larger tumors containing more clones that are resistant to immune checkpoint inhibitors. To overcome this heterogeneity, studies have investigated the synergic benefits of combination therapy for advanced HCC (40). Lenvatinib combined with pembrolizumab or bevacizumab with atezolizumab demonstrated unprecedented objective response rates $(41,42)$. These reports suggest that the resistance to immune checkpoint inhibitors can be overcome by the combination of drugs with different mechanisms of action. Future studies will demonstrate the role of DWI in predicting the responses of various immune and combination therapies.

In conclusion, our study demonstrated for the first time that patients with unresectable HCC with a pretreatment tumor-toliver ADC ratio $<0.741$ showed a favorable intrahepatic response to HAIC. Therefore, diffusion-weighted MR imaging can play a critical role as a predictor of response to cisplatin-based HAIC in unresectable HCC. 


\section{DATA AVAILABILITY STATEMENT}

The original contributions presented in the study are included in the article/Supplementary Material. Further inquiries can be directed to the corresponding authors.

\section{ETHICS STATEMENT}

Ethical approval was obtained from the Institutional Review Board of Seoul St. Mary's Hospital (KC19RESI0912). The patients/participants provided their written informed consent to participate in this study.

\section{AUTHOR CONTRIBUTIONS}

PS: study design, data collection, data analysis, data interpretation, manuscript writing, and manuscript approval. MC: data collection, data analysis, data interpretation, manuscript writing, and

\section{REFERENCES}

1. Yang JD, Hainaut P, Gores GJ, Amadou A, Plymoth A, Roberts LR. A global view of hepatocellular carcinoma: trends, risk, prevention and management. Nat Rev Gastroenterol Hepatol (2019) 16:589-604. doi: 10.1038/s41575-0190186-y

2. Sung PS, Yang K, Bae SH, Oh JS, Chun HJ, Nam HC, et al. Reduction of Intrahepatic Tumour by Hepatic Arterial Infusion Chemotherapy Prolongs Survival in Hepatocellular Carcinoma. Anticancer Res (2019) 39:3909-16. doi: 10.21873/anticanres. 13542

3. Sung PS, Park HL, Yang K, Hwang S, Song MJ, Jang JW, et al. (18)Ffluorodeoxyglucose uptake of hepatocellular carcinoma as a prognostic predictor in patients with sorafenib treatment. Eur J Nuclear Med Mol Imaging (2018) 45:384-91. doi: 10.1007/s00259-017-3871-5

4. Ogasawara S, Ooka Y, Koroki K, Maruta S, Kanzaki H, Kanayama K, et al. Switching to systemic therapy after locoregional treatment failure: Definition and best timing. Clin Mol Hepatol (2020) 26:155-62. doi: 10.3350/ cmh.2019.0021n

5. Sung PS, Jang JW, Lee J, Lee SK, Lee HL, Yang H, et al. Real-World Outcomes of Nivolumab in Patients With Unresectable Hepatocellular Carcinoma in an Endemic Area of Hepatitis B Virus Infection. Front Oncol (2020) 10:1043. doi: 10.3389/fonc.2020.01043

6. Choi JH, Chung WJ, Bae SH, Song DS, Song MJ, Kim YS, et al. Randomized, prospective, comparative study on the effects and safety of sorafenib vs. hepatic arterial infusion chemotherapy in patients with advanced hepatocellular carcinoma with portal vein tumor thrombosis. Cancer Chemother Pharmacol (2018) 82:469-78. doi: 10.1007/s00280-018-3638-0

7. He M, Li Q, Zou R, Shen J, Fang W, Tan G, et al. Sorafenib Plus Hepatic Arterial Infusion of Oxaliplatin, Fluorouracil, and Leucovorin vs Sorafenib Alone for Hepatocellular Carcinoma With Portal Vein Invasion: A Randomized Clinical Trial. JAMA Oncol (2019) 5:953-60. doi: 10.1001/ jamaoncol.2019.0250

8. Lin CC, Hung CF, Chen WT, Lin SM. Hepatic Arterial Infusion Chemotherapy for Advanced Hepatocellular Carcinoma with Portal Vein Thrombosis: Impact of Early Response to 4 Weeks of Treatment. Liver Cancer (2015) 4:228-40. doi: 10.1159/000367737

9. Kim TH, Kim SY, Tang A, Lee JM. Comparison of international guidelines for noninvasive diagnosis of hepatocellular carcinoma: 2018 update. Clin Mol Hepatol (2019) 25:245-63. doi: 10.3350/cmh.2018.0090

10. Choi SY, Kim SH, Park CK, Min JH, Lee JE, Choi YH, et al. Imaging Features of Gadoxetic Acid-enhanced and Diffusion-weighted MR Imaging for manuscript approval. HY, HC, JJ, JC, SY, J-IC, YL, and SB: data interpretation and manuscript approval. All authors contributed to the article and approved the submitted version.

\section{FUNDING}

This research was supported by the Basic Science Research Program through the National Research Foundation of Korea (NRF) (NRF-2019R1I1A1A01059642 to PS and NRF2020R1A2C3011569 to SB). This work was also supported by Research Fund of Seoul St. Mary's Hospital, The Catholic University of Korea (PS).

\section{SUPPLEMENTARY MATERIAL}

The Supplementary Material for this article can be found online at: https://www.frontiersin.org/articles/10.3389/fonc.2020. 600233/full\#supplementary-material

Identifying Cytokeratin 19-positive Hepatocellular Carcinoma: A Retrospective Observational Study. Radiology (2018) 286:897-908. doi: 10.1148/radiol.2017162846

11. Cho ES, Choi JY. MRI features of hepatocellular carcinoma related to biologic behavior. Korean J Radiol (2015) 16:449-64. doi: 10.3348/kjr.2015.16.3.449

12. Messina C, Bignone R, Bruno A, Bruno A, Bruno F, Calandri M, et al. Diffusion-Weighted Imaging in Oncology: An Update. Cancers (Basel) (2020) 12:1493. doi: $10.3390 /$ cancers 12061493

13. Taouli B, Koh DM. Diffusion-weighted MR imaging of the liver. Radiology (2010) 254:47-66. doi: 10.1148/radiol.09090021

14. Saito K, Tajima Y, Harada TL. Diffusion-weighted imaging of the liver: Current applications. World J Radiol (2016) 8:857-67. doi: 10.4329/ wir.v8.i11.857

15. Mannelli L, Kim S, Hajdu CH, Babb JS, Taouli B. Serial diffusion-weighted MRI in patients with hepatocellular carcinoma: Prediction and assessment of response to transarterial chemoembolization. Prelim Experience Eur J Radiol (2013) 82:577-82. doi: 10.1016/j.ejrad.2012.11.026

16. Sahin H, Harman M, Cinar C, Bozkaya H, Parildar M, Elmas N. Evaluation of treatment response of chemoembolization in hepatocellular carcinoma with diffusion-weighted imaging on 3.0-T MR imaging. J Vasc Interv Radiol (2012) 23:241-7. doi: 10.1016/j.jvir.2011.08.030

17. Vandecaveye V, Michielsen K, De Keyzer F, Laleman W, Komuta M, Op de beeck K, et al. Chemoembolization for hepatocellular carcinoma: 1-month response determined with apparent diffusion coefficient is an independent predictor of outcome. Radiology (2014) 270:747-57. doi: 10.1148/ radiol.13130591

18. e.e.e. European Association for the Study of the Liver. Electronic address, and L. European Association for the Study of the, EASL Clinical Practice Guidelines: Management of hepatocellular carcinoma. J Hepatol (2018) 69:182-236. doi: 10.1016/j.jhep.2018.03.019

19. Heimbach JK, Kulik LM, Finn RS, Sirlin CB, Abecassis MM, Roberts LR, et al. AASLD guidelines for the treatment of hepatocellular carcinoma. Hepatology (2018) 67:358-80. doi: 10.1002/hep.29086

20. Deng J, Miller FH, Rhee TK, Sato KT, Mulcahy MF, Kulik LM, et al. Diffusionweighted MR imaging for determination of hepatocellular carcinoma response to yttrium-90 radioembolization. J Vasc Interv Radiol (2006) 17:1195-200. doi: 10.1097/01.RVI.0000227234.81718.EB

21. Hilmi M, Neuzillet C, Calderaro J, Lafdil F, Pawlotsky JM, Rousseau B. Angiogenesis and immune checkpoint inhibitors as therapies for hepatocellular carcinoma: current knowledge and future research directions. J Immunother Cancer (2019) 7:333. doi: 10.1186/s40425-019-0824-5 
22. Lim KS. Diffusion-weighted MRI of hepatocellular carcinoma in cirrhosis. Clin Radiol (2014) 69:1-10. doi: 10.1016/j.crad.2013.07.022

23. Llovet JM, Ricci S, Mazzaferro V, Hilgard P, Gane E, Blanc JF, et al. Sorafenib in advanced hepatocellular carcinoma. N Engl J Med (2008) 359:378-90. doi: 10.1056/NEJMoa0708857

24. Lewin M, Fartoux L, Vignaud A, Arrive L, Menu Y, Rosmorduc O. The diffusion-weighted imaging perfusion fraction $\mathrm{f}$ is a potential marker of sorafenib treatment in advanced hepatocellular carcinoma: a pilot study. Eur Radiol (2011) 21:281-90. doi: 10.1007/s00330-010-1914-4

25. Do RK, Chandarana H, Felker E, Hajdu CH, Babb JS, Kim D, et al. Diagnosis of liver fibrosis and cirrhosis with diffusion-weighted imaging: value of normalized apparent diffusion coefficient using the spleen as reference organ. AJR Am J Roentgenol (2010) 195:671-6. doi: 10.2214/AJR.09.3448

26. Park SH, Moon WK, Cho N, Song IC, Chang JM, Park IA, et al. Diffusionweighted MR imaging: pretreatment prediction of response to neoadjuvant chemotherapy in patients with breast cancer. Radiology (2010) 257:56-63. doi: 10.1148/radiol.10092021

27. Park IK, Yu JS, Cho ES, Kim JH, Chung JJ. Apparent diffusion coefficient of hepatocellular carcinoma on diffusion-weighted imaging: Histopathologic tumor grade versus arterial vascularity during dynamic magnetic resonance imaging. PloS One (2018) 13:e0197070. doi: 10.1371/journal.pone.0197070

28. Boussouar S, Itti E, Lin SJ, Decaens T, Evangelista E, Chiaradia M, et al. Functional imaging of hepatocellular carcinoma using diffusion-weighted MRI and (18)F-FDG PET/CT in patients on waiting-list for liver transplantation. Cancer Imaging Off Publ Int Cancer Imaging Soc (2016) 16:4. doi: 10.1186/s40644-016-0062-8

29. Yang H, Woo HY, Lee SK, Han JW, Jang B, Nam HC, et al. A comparative study of sorafenib and metronomic chemotherapy for Barcelona Clinic Liver Cancer-stage C hepatocellular carcinoma with poor liver function. Clin Mol Hepatol (2017) 23:128-37. doi: 10.3350/cmh.2016.0071

30. Faivre S, Rimassa L, Finn RS. Molecular therapies for HCC: Looking outside the box. J Hepatol (2020) 72:342-52. doi: 10.1016/j.jhep.2019.09.010

31. Kudo M. Lenvatinib May Drastically Change the Treatment Landscape of Hepatocellular Carcinoma. Liver Cancer (2018) 7:1-19. doi: 10.1159/000 487148

32. Kudo M, Finn RS, Qin S, Han K-H, Ikeda K, Piscaglia F, et al. Lenvatinib versus sorafenib in first-line treatment of patients with unresectable hepatocellular carcinoma: a randomised phase 3 non-inferiority trial. Lancet (2018) 391:1163-73. doi: 10.1016/S0140-6736(18)30207-1

33. Yu LX, Ling Y, Wang HY. Role of nonresolving inflammation in hepatocellular carcinoma development and progression. NPJ Precis Oncol (2018) 2:6. doi: 10.1038/s41698-018-0048-z
34. Ringelhan M, Pfister D, O'Connor T, Pikarsky E, Heikenwalder M. The immunology of hepatocellular carcinoma. Nat Immunol (2018) 19:222-32. doi: 10.1038/s41590-018-0044-z

35. Sachdeva M, Chawla YK, Arora SK. Immunology of hepatocellular carcinoma. World J Hepatol (2015) 7:2080-90. doi: 10.4254/wjh.v7.i17.2080

36. Sung PS, Jang JW. Natural Killer Cell Dysfunction in Hepatocellular Carcinoma: Pathogenesis and Clinical Implications. Int J Mol Sci (2018) 19:3648. doi: 10.20944/preprints201810.0450.v1

37. Finn RS, Ryoo BY, Merle P, Kudo M, Bouattour M, Lim HY, et al. Pembrolizumab As Second-Line Therapy in Patients With Advanced Hepatocellular Carcinoma in KEYNOTE-240: A Randomized, Double-Blind, Phase III Trial. J Clin Oncol (2020) 38:193-202. doi: 10.1200/JCO.19.01307

38. Zhu AX, Finn RS, Edeline J, Cattan S, Ogasawara S, Palmer D, et al. Pembrolizumab in patients with advanced hepatocellular carcinoma previously treated with sorafenib (KEYNOTE-224): a non-randomised, open-label phase 2 trial. Lancet Oncol (2018) 19:940-52. doi: 10.1016/S1470-2045(18)30351-6

39. El-Khoueiry AB, Sangro B, Yau T, Crocenzi TS, Kudo M, Hsu C, et al. Nivolumab in patients with advanced hepatocellular carcinoma (CheckMate 040): an openlabel, non-comparative, phase $1 / 2$ dose escalation and expansion trial. Lancet (2017) 389:2492-502. doi: 10.1016/S0140-6736(17)31046-2

40. Cheng AL, Hsu C, Chan SL, Choo SP, Kudo M. Challenges of combination therapy with immune checkpoint inhibitors for hepatocellular carcinoma. J Hepatol (2020) 72:307-19. doi: 10.1016/j.jhep.2019.09.025

41. Finn RS, Qin S, Ikeda M, Galle PR, Ducreux M, Kim TY, et al. Atezolizumab plus Bevacizumab in Unresectable Hepatocellular Carcinoma. N Engl J Med (2020) 382:1894-905. doi: 10.1056/NEJMoa1915745

42. Finn RS, Ikeda M, Zhu AX, Sung MW, Baron AD, Kudo M, et al. Phase Ib Study of Lenvatinib Plus Pembrolizumab in Patients With Unresectable Hepatocellular Carcinoma. J Clin Oncol (2020) 38:2960-70. doi: 10.1200/ JCO.20.00808

Conflict of Interest: The authors declare that the research was conducted in the absence of any commercial or financial relationships that could be construed as a potential conflict of interest.

Copyright (๑) 2020 Sung, Choi, Yang, Lee, Chun, Jang, Choi, Yoon, Choi, Lee and Bae. This is an open-access article distributed under the terms of the Creative Commons Attribution License (CC BY). The use, distribution or reproduction in other forums is permitted, provided the original author(s) and the copyright owner(s) are credited and that the original publication in this journal is cited, in accordance with accepted academic practice. No use, distribution or reproduction is permitted which does not comply with these terms. 